\title{
Risk Factor Profiles in Patients with Different Clinical Manifestations of Venous Thromboembolism: A Focus on the Factor V Leiden Mutation
}

\author{
Bert Manten 1,2, Rudi G. J. Westendorp ${ }^{12}$, Ted Kosterl, Pieter H. Reitsma3 \\ Frits R. Rosendaal ${ }^{1,3}$
}

From the 'Departments of Clinical Epidemiology, ${ }^{2}$ General Internal Medicine and ${ }^{3}$ Hematology, Leiden, The Netherlands

\section{Summary}

Background. Patients with venous thromboembolic disease may present with different clinical manifestations. Factor $V$ Leiden mutation leading to resistance to activated protein $\mathrm{C}$ is associated with a sevenfold increased risk for presenting with deep-vein thrombosis. It is not yet established whether carriers of the mutation have a similarly increased risk for manifesting with pulmonary embolism.

Methods. From an Anticoagulation Clinic monitoring coumarin therapy, a consecutive series of patients with a first thromboembolic event (objectively proven by current radiological methods) were enrolled. All patients were interviewed and blood was drawn for genotyping. From the hospital charts and the personal interview, information was obtained on acquired risk factors and the signs and symptoms on hospital admission.

Results. 45 patients presented with symptoms of pulmonary embolism only, 211 had only symptoms of deep-vein thrombosis whereas 23 had clinical features of both. In about half of the patients acquired risk factors for venous thromboembolism were present which did not differ between the three groups of patients. Recent surgery had been performed more often in patients presenting with pulmonary embolism than in other patients $(33.3 \%$ vs. $18.5 \%, \mathrm{p}<0,05)$. Factor V Leiden was present in $9 \%$ of the patients presenting with pulmonary embolism (relative risk: $3.395 \% \mathrm{CI}: 1.0-10.6$ ) and $17 \%$ of the patients presenting with deep-vein thrombosis (relative risk: $6.995 \% \mathrm{CI}$ : 3.6-12.8). The prevalence of factor $\mathrm{V}$ Leiden was intermediate in patients with both clinical characteristics.

Conclusion. These data suggest that patients with venous thromboembolism have different clinical presentation depending on the risk factor profile. Factor V Leiden may preferentially lead to manifest deep-vein thrombosis. Differences in structure of venous thrombi could underlie differences in embolic tendency.

\section{Introduction}

Patients with venous thromboembolic disease may present with different clinical manifestations. Pulmonary embolism without signs or symptoms of the underlying deep-vein thrombosis is one end of the spectrum while deep-vein thrombosis without clinical features of pulmonary embolism represents the other. At present, it is not known whether there are specific factors that determine how venous thromboembolism becomes clinically apparent.

Correspondence to: R. G. J. Westendorp, Dept. Clinical Epidemiology, Leiden University Hospital, DOP-45, P.O. Box 9600, 2300 RC Leiden, The Netherlands - Tel.: +31715264037; FAX Number: +31715248122
Intensive clinical, epidemiological and laboratory research has led to the identification of several hereditary and acquired conditions predisposing to deep-vein thrombosis (1). The most common genetic risk factor is the recently described factor $V$ Leiden, a single point mutation in the factor $V$ gene (2), causing resistance to activated protein $C(3)$, which increases the risk for deep-venous thrombosis about seven-fold (4).

Since venous thromboembolism is considered as one single disease entity, it is assumed that the hereditary and acquired risk factors in patients presenting with deep-vein thrombosis, are similar to those in patients presenting with pulmonary embolism. The aim of the present study is to validate this assumption by a comparison of the risk factor profiles in patients with different clinical manifestations of venous thromboembolism. As factor $V$ Leiden is the most frequent inherited disorder of the anticoagulant system, we will focus on the association of this factor with the different clinical presentations of venous thromboembolic disease.

\section{Methods}

Setting

In the Netherlands, all patients requiring oral anticoagulant treatment are referred to an Anticoagulation Clinic for outpatient monitoring, each of which serves a well defined geographical area (5). To investigate the distribution of risk factors in patients with different clinical manifestations of venous thromboembolism, we divided the patients referred to the Leiden Anticoagulation Clinic in three study groups. In the first group, patients with deep-vein thrombosis without signs or symptoms of pulmonary embolism were included. Patients with pulmonary embolism without any sign or symptom of the underlying deep-vein thrombosis were assigned to the second group. The third group consisted of patients who presented with the clinical manifestations of both deep-vein thrombosis and pulmonary embolism.

\section{Patients with Symptoms of Deep-Vein Thrombosis only}

In a previous case- control study, embedded in three Anticoagulation Clinics, consecutive patients with a first episode of deep-vein thrombosis had been included. Patients over 70 years of age and patients with a malignancy were excluded. Each patient was interviewed in the outpatient department after a median time of 19 months since diagnosis. During this visit, blood was drawn to determine the presence of the factor V Leiden mutation by genotyping according to established laboratory procedures, described in detail elsewhere (2). Results of this study in patients with deep-vein thrombosis have been reported earlier $(4,6)$.

In the present analysis, only patients treated in the Leiden Anticoagulation Clinic were included of whom detailed information on their hospital admission was available. These patients had been diagnosed by ultrasound or venography in the period January 1988-December 1992. Available data included the clini- 
cal history, physical examination, imaging techniques and the disease course. From the medical records and the personal interview, we obtained information on the presence or absence of acquired risk factors, defined as recent surgery ( $<2$ months before admission), hospitalisation without surgery, prolonged bedrest ( $>2$ weeks within 30 days of the admission date) at home, post partum periods (within 30 days of the admission date), and recent trauma ( $<3$ weeks) of the involved $\operatorname{limb}(1,7)$.

\section{Patients with Symptoms of Pulmonary Embolism only}

In the period 1988-1989, a consecutive series of patients who had been treated in the Leiden Anticoagulation Clinc for pulmonary embolism without clinical signs or symptoms of deep-vein thrombosis were considered. For these patients, we reviewed the evidence on which the diagnosis of pulmonary embolism was based. Only patients with an objectively confirmed diagnosis, i.e. a positive pulmonary angiography or a (ventilation) perfusion lungscan with defects indicating high- probability for pulmonary embolism were included in the present analysis. Of these patients, laboratory procedures and collection of relevant information were establıshed in the same way as for patients with deep-vein thrombosis mentioned above.

\section{Patients with Symptoms of both Deep-Vein Thrombosis and Pulmonary Embolism}

In all patients with deep-vein thrombosis included in the first group, the clinical history was evaluated for signs or symptoms indicating the presence of a concomitant pulmonary embolism. Since these patients already suffered from an objectively confirmed deep-vein thrombosis, rendering a high prior probability of chest symptoms to be due to pulmonary embolism, the diagnostic criteria for pulmonary embolism mentioned above were extended. Patients with paroxysms or acute onset of dyspnoe, chest pain of pleuritic nature or a pleural friction rub on physical examination were also considered having a proven pulmonary embolism when imaging techniques were not available. These patients presenting with deep-vein thrombosis complicated by a clinically manifest pulmonary embolism, were analysed as a separate group.

\section{Control Subjects}

To compare the distribution of risk factors for venous thromboembolism in the three patient groups to the normal population and to calculate odds ratios as a measure of relative risk, a control group was included. It consisted of the control subjects of the original case control study, who were individuals without a history of venous thromboembolism (4). In these individuals, the presence of acquired risk factors was evaluated by use of a questionnaire, and blood samples for factor $\mathrm{V}$ Leiden determination were drawn in the same session.

\section{Statistical Analysis}

Between the various study groups, the distribution of acquired risk factors and factor $V$ Leiden were compared using the $\chi^{2}$-statistic. Exposure odds ratios were calculated as estimates of relative risks for thromboembolic disease. Odds ratios were adjusted for age and sex in a logistic regression model. 95\%Confidence intervals $(95 \% \mathrm{CI})$ for the odds ratios were derived from the standard error of the coefficient of the model.

\section{Results}

A total of 55 patients were identified who had been treated with oral anticoagulants for a diagnosis of pulmonary embolism without signs or symptoms of deep-vein thrombosis. On revision, ten of these patients were excluded because the diagnosis was not objectively proven, leaving 45 patients for analysis. Of the 234 patients with deep-vein thrombosis, $23(10 \%)$ were diagnosed with concomitant pulmonary
Table 1 Age and sex distributions of patients classified on their clinical presentation

\begin{tabular}{lllll}
\hline & PE only & DVT with PE & DVT only & controls \\
\hline number & 45 & 23 & 211 & 474 \\
mean age (yrs) & 456 & 413 & 41.2 & 441 \\
Sex $(F / M)$ & $13 / 32$ & $10 / 13$ & $121 / 90$ & $272 / 202$ \\
\hline
\end{tabular}

PE only, patients with only symptoms of pulmonary embolism

DVT only, patients with only symptoms of deep-vein thrombosis

DVT with PE, patients with symptoms of both deep-vein thrombosis and pulmonary

embolism

embolism during hospital admission. All other 211 patients lacked signs or symptoms indicating pulmonary involvement. Age and sex distributions of the study groups are shown in Table 1 . The female/male ratio in the patients presenting with pulmonary embolism was significantly lower than in the other study groups.

The risk factor profiles of patients presenting with pulmonary embolism only and patients with deep-vein thrombosis with and without concomitant pulmonary embolism are presented in Table 2 . The prevalence of the factor V Leiden mutation was lowest in patients with symptoms of pulmonary embolism only and highest in patients with deep-vein thrombosis only, whereas the prevalence was in-between in patients with symptoms of both. When confined to the subgroups of patients without acquired risk factors, factor V Leiden was present in $22.7 \%$ (25 out of 110) of the patients presenting with deep-vein thrombosis

Table 2 Comparison of risk factors between patients classified on their clinical presentation

\begin{tabular}{|c|c|c|c|}
\hline & PE only & DVT with PE & DVT only \\
\hline Risk factor & $(n=45)$ & $(n=23)$ & $(n=211)$ \\
\hline none & $23(51 \% \%)$ & $12(52.2 \%)$ & $85(403 \%)$ \\
\hline factor V Leiden & $4(89 \%)$ & $3(130 \%)$ & $37(175 \%)$ \\
\hline acquired & $19(422 \%)$ & $9(391 \%)$ & $101(479 \%)$ \\
\hline recent surgery & 15 & 3 & 39 \\
\hline hospitalization & 1 & 1 & 11 \\
\hline immobilization & - & - & 6 \\
\hline post partum & 1 & 1 & 15 \\
\hline trauma & 2 & 4 & 30 \\
\hline
\end{tabular}

PE only patients with only symptoms of pulmonary embolism,

DVT only patients with only symptoms of deep-vein thrombosis

DVT with PE patients with symptoms of both deep-vein thrombosis and pulmonary

embolism

Totals can add up to more than $100 \%$ since patients can have more than one risk factor 
Table 3 Relative risks of factor V Leiden in patients with pulmonary embolism and deep-vein thrombosis

\begin{tabular}{lccc}
\hline & \multicolumn{2}{c}{ factor V Leiden } & \\
\cline { 2 - 3 } Group $(n)$ & present & absent & RR $(95 \%$ CI) \\
\hline controls (474) & 14 & 460 & 1 \\
PE only (45) & 4 & 41 & $3.3(1.0-10.6)$ \\
DVT only (211) & 37 & 174 & $6.9(3.6-12.8)$ \\
\hline
\end{tabular}

PE only: patients with only symptoms of pulmonary embolism

DVT only: patients with only symptoms of deep-vein thrombosis

$95 \% \mathrm{Cl}: 95 \%$ confidence interval.

Relative risks (RR) were estimated with exposure odds ratios and adjusted for age and sex by logistic regression.

only, and in $11.5 \%$ ( 3 out of 26 ) of the patients with pulmonary embolism only $\left(\chi^{2}=1.12, \mathrm{df}=1, \mathrm{p}=0.28\right)$. Three patients presenting deep-vein thrombosis and one patiënt with clinical features of both deep-vein thrombosis and pulmonary embolism were homozygous carriers of the trait. Neither in the control group nor in the patients presenting with pulmonary embolism, homozygous carriers could be identified.

Recent surgery had been performed in $33.3 \%$ (15 out of 45) of the patients presenting with pulmonary embolism only and in $18.5 \%$ (39 out of 211) of the patients presenting with deep-vein thrombosis only $\left(\chi^{2}=4.9, \mathrm{df}=1, \mathrm{p}=0.03\right)$. In patients with clinical features of both this percentage was 13.0 ( 3 out of 23 ).

To further analyze the contribution of the factor $V$ Leiden mutation to the different clinical manifestations of venous thromboembolic disease, exposure odds ratios were calculated (Table 3). Factor V Leiden increased the risk for pulmonary embolism three-fold $(\mathrm{OR}=3.3,95 \% \mathrm{CI} 1.0-10.6)$. The risk for deep-vein thrombosis was seven-fold $(\mathrm{OR}=6.9,95 \% \mathrm{CI} 3.6-12.8)$ increased in the carriers of factor V Leiden.

\section{Discussion}

The overall percentage of patients with acquired risk factors for venous thromboemblism did not differ between the three study groups. This supports the findings in a recently reported series of patients with manifest deep-vein thrombosis in which also no association was found between various acquired risk factors and the occurrence of concomitant pulmonary embolism (7). These authors, however, did not investigate hereditary disorders predisposing to venous thromboembolism, nor did they include patients presenting with pulmonary embolism without signs or symptoms of deep-vein thrombosis.

In our study, recent surgery had been performed in a higher percentage of patients presenting with pulmonary embolism. There was no difference in the frequency of recent surgery between patients presenting with deep-vein thrombosis with or without clinically manifest pulmonary embolism. Although there is no obvious explanation yet, this finding demonstrates that significant differences in risk factors emerge between patients with venous thromboembolism when they are classified on their clinical manifestations. This notion is enforced by the remarkable sex difference between the study groups.
We expected that factor V Leiden would be more prevalent in the patients presenting with pulmonary embolism since resistance to activated protein $\mathrm{C}$ may lead to more extensive venous thrombosis and a subsequently higher risk for pulmonary embolism. Contrary to this expectation, the prevalence of factor $V$ Leiden was lower in patients with the clinical presentation of pulmonary embolism only. The age and sex adjusted risk for presenting with pulmonary embolism in carriers of the trait was "only" three-fold increased in comparison with the seven-fold increased risk for deep-vein thrombosis. Partly in line with these observations are the results of a recently reported study investigating the prevalence of activated protein $C$ (APC) resistance in an unselected series of patients presenting with pulmonary embolism without clinical signs or symptoms of deep-vein thrombosis (11). In patients with an objectively confirmed diagnosis, the prevalence of APC resistance was $5.5 \%$ which was only slightly different from the prevalence of $4.0 \%$ found in the patients in whom the diagnosis was ruled out. In this study, however, no DNA analysis was performed to confirm the presence of factor V Leiden in APC resistant patients and unfortunately no subdivision was made as to underlying disease.

It has been shown that thrombi confined to the calf veins pose limited embolic risk $(8,9,10)$. How the location of a thrombus affects its embolic potential is unknown, although it is suggested that size is an important determinant (12). In analogy, it may be postulated that the various etiologic mechanisms (e.g. genetic background and stasis of blood flow) lead to venous thrombosis with different embolic and obstructive potential, similar to the differences in red and white arterial thrombosis. Our findings support the hypothesis that venous thrombi caused by various etiologic mechanisms have different structure and factor V Leiden leads to thrombi with smaller embolic risk.

An objection to our findings may be diagnostic misclassification. It is unlikely, however, that this can explain our observations since only patients who satisfied current, stringent, radiological criteria for pulmonary embolism were included in the study, and the positive predictive value of a high probability result of a ventilation-perfusion lungscan is almost $90 \%(12,13)$. A substantial number of patients, classified as having deep-vein thrombosis only, will have had silent pulmonary embolism. Also, a high proportion of patients presenting with pulmonary embolism, will have had asymptomatic deep-vein thrombosis when investigated by venography. From a methodological point of view this will only have deflated the risk differences between the study groups and does not argue against the hypothesis. Furthermore, there are relevant arguments to support our objective to compare risk factor profiles in patients with different clinical manifestations of venous thromboembolism. Clinically manifest pulmonary embolism is still considered the most serious (i.e. lethal) presentation of venous thromboembolism. It also remains an absolute indication for anticoagulant treatment, whereas some cases of deep- vein thrombosis (e.g. confined to the calf veins) will not be treated. The concerns about the impact of risk factors on mortality and morbidity of venous thromboembolic disease are directly related to the possible occurrence of a clinically manifest pulmonary embolism. The findings in our study suggest that results from studies on risk factors in patients with deep-vein thrombosis may not automatically be applied to patients presenting with pulmonary embolism.

A high percentage of patients in our study groups did not have an identifiable risk factor for the occurrence of venous thromboembolic disease. As the factor V Leiden mutation is the most frequent inherited disorder of the anticoagulant system, it is unlikely that a large proportion of patients without risk factors will have had other hereditary thrombotic disorders (i.e. protein $C, S$ or antithrombin deficiencies). In 
fact, only one patient presenting with pulmonary embolism only was identified as protein $\mathrm{C}$ deficient by DNA analysis. It might be possible, however, that future research will identify new coagulation disorders in these patients.

The recent identification of factor V Leiden and its high prevalence in the general population has provided new elements for the discussion on prevention and management of venous thromboembolism $(15,16$, 17). From a clinical and public health perspective, the concern should focus on the occurrence of pulmonary embolism as a potentially lethal manifestation of venous thromboembolic disease $(1,16)$. The present study indicates that risk factors may have different impact on venous thrombotic and embolic disease.

\section{References}

1. Weinmann EE, Salzman EW. Deep- vein thrombosis. N Engl J Med 1994; 331: 630-41.

2. Bertina RM, Koeleman BPC, Koster T, Rosendaal FR, Dirven RJ, de Ronde $\mathrm{H}$, van der Velden PA, Reitsma PH. Mutation in blood coagulation associated with resistance to activated protein C. Nature 1994; 369: 64-7.

3. Dahlbäck B, Carlsson M, Svensson PJ. Familial thrombophilia due to a previously unrecognized mechanism characterized by poor anticoagulant response to activated protein $\mathrm{C}$ : prediction of a cofactor to activated protein C. Proc Natl Acad Sci USA 1993; 90: 1004-8.

4. Koster T, Rosendaal FR, de Ronde H, Briët E, Vandenbroucke JP, Bertina RM. Venous thromboembolism due to poor anticoagulant response to activated protein C: Leiden Thrombophilia Study. Lancet 1993; 342: 1503-6.

5. Loeliger EA, van Dijk- Wierda CA, van den Besselaar AMHP, Broekmans $A W$, Roos J. Anticoagulant therapy and the risk of bleeding; organizational infrastructure. In: Meade TW, ed. Anticoagulants and myocardial infarction; a reappraisal. Chicester, John Wiley and sons, 1984: 157.

6. Rosendaal FR, Koster T, Vandenbroucke JP, Reitsma PH. High risk of thrombosis in patients homozygous for factor $\mathrm{V}$ Leiden (activated protein $\mathrm{C}$ resistance). Blood 1995; 85: 1504-8.
7. Salzman EW, Hirsh J. The epidemiology, pathogenesis, and natural history of venous thrombosis. In: Hemostasis and Thrombosis: basic principles and practice. Colman RW, Hirsh J, Marder VJ, Salzman EW, eds. 3rd ed. Philadelphia; J B Lippincott 1994: 1275-96.

8. Monréal M, Ruiz J, Olazabal A, Arias A, Roca J. Deep venous thrombosis and the risk of pulmonary embolism. Chest 1992; 102: 677-81.

9. Moser KM, Le Moine JR. Is embolic risk conditioned by location of deep venous thrombosis? Ann Int Med 1981; 94: 439-44.

10. Philbrick JT, Becker DM. Calf deep venous thrombosis: a wolf in sheep's clothing? Arch Intern Med 1988; 148: 2131-8.

11. Desmarais S, de Moerloose P, Reber G, Minazio A, Bounameaux H. Resistance to activated protein $C$ in an unselected population of patients with pulmonary embolism. Lancet 1996; 347: 1374-5.

12. Hull RD, Hirsh J, Carter CJ, Jay RM, Dodd PE, Ockelford PA, Coates G, Gill GJ, Turpie AG, Doyle DJ, Buller HR, Raskob GE. Pulmonary angiography, ventilation lung scanning, and venography for clinically suspected pulmonary embolism with abnormal perfusion lung scan. Ann Int Med 1983; 98: 891-9.

13. Quinn DA, -Thompson T, Terrin ML, Thrall $\mathrm{JH}$, Athanasoulis CA, McKusick KA, Stein PD, Hales CA. A prospective investigation of pulmonary embolism in women and men. JAMA 1992; 268: 1689-96.

14. Hull RD, Hirsh J, Carter CJ, Raskob GE, Gill GJ, Jay RM, Leclerc JR, David M, Coates G. Diagnostic value of ventilation- perfusion lung scanning in patients with suspected pulmonary embolism. Chest 1985; 88: 819-28.

15. Dahlbäck B. Inherited thrombophilia: resistance to activated protein $C$ as a pathogenic factor of venous thromboembolism. Blood 1995; 85: 607-14.

16. Ridker PM, Hennekens CH, Lindpaintner K, Stampfer ML, Eisenberg PR, Miletich JP. Mutation in the gene coding for coagulation factor $\mathrm{V}$ and the risk of myocardial infarction, stroke, and venous thromboembolism in apparently healthy men. N Engl J Med 1995; 332: 912-7.

17. Vandenbroucke JP, Koster T, Briët E, Reitsma PH, Bertina RM, Rosendaal FR. Increased risk of venous thrombosis in oral- contraceptive users who are carrier of factor V Leiden mutation. Lancet 1994; 344: 1453-7.

Received February 22, 1996 Accepted after resubmission June 19, 1996 